\title{
CDA Inhibitor E7727/Decitabine Combination Agent ASTX727
}

National Cancer Institute

\section{Source}

National Cancer Institute. CDA Inhibitor E7727/Decitabine Combination Agent ASTX727.

NCI Thesaurus. Code C115102.

An orally available combination agent containing the cytidine deaminase (CDA) inhibitor E7727 and the cytidine antimetabolite decitabine, with potential antineoplastic activity. Upon oral administration of ASTX727, the CDA inhibitor E7727 binds to and inhibits CDA, an enzyme primarily found in the gastrointestinal $(\mathrm{Gl})$ tract and liver that catalyzes the deamination of cytidine and cytidine analogs. This prevents the breakdown of decitabine, increases its bioavailability and efficacy while decreasing Gl toxicity due to the administration of lower doses of decitabine. Decitabine exerts its antineoplastic activity through the incorporation of its triphosphate form into DNA, which inhibits DNA methyltransferase and results in hypomethylation of DNA. This interferes with DNA replication and decreases tumor cell growth. 